\section{MS46-O5 An Enhanced Hirshfeld Test - Validating Atomic Vibrations in Crystal Structures}

Jens Luebben ${ }^{1}$, Birger Dittrich ${ }^{1}$, George Sheldrick ${ }^{2}$

1. Heinrich-Heine-University Duesseldorf, Germany

2. Georg-August-University Goettingen, Germany

email: jens.luebben@chemie.uni-goettingen.de

The current routine procedure for publishing crystal structures of small molecules includes structure validation via the the IUCr CheckCIF service. [1] Part of that procedure is the Hirshfeld test [2] which ensures that the atomic vibrations, encoded as Atomic Displacement Parameters (ADPs), are physically reasonable. While the Hirshfeld test is fast and efficient at finding potential problems in the structural model, there exist a number of borderline cases where the test is not able to find implausible ADPs. In other cases the CheckCIF routine might raise alerts where the structure is actually perfectly fine.

An improved procedure will be presented which aims to address these problems. The procedure is based on the Hirshfeld test and inspired by the 'RIGU' restraint from the Shelxl [3] program. This is achieved by analyzing the ADPs characteristics in all three spacial dimensions instead of only validating its expansion in bond direction and taking the atomic masses applied to a harmonic oscillator model into account.

This procedure achieves its goal of finding problematic ADPs in cases that are not found by the traditional Hirshfeld test and is able to reasonably evaluate the ADPs of atoms with significantly different atomic masses e.g. carbon - hydrogen bonds in neutron diffraction studies.

[1] Spek Acta Cryst (2015). C71, 9-18.

[2] Rosenfield et al., Acta Cryst (1978). A34, 828-829.

[3] Thorn et al., Acta Cryst. (2012). A68, 448-451.

Keywords: Validation, ADPs

\section{MS47 Teaching \& Education}

Chairs: Helen Stoeckli-Evans, Howard Flack

MS47-01 It is never too early, or too late to start

Elena V. Boldyreva ${ }^{1,2}$, Sergey G. Arkhipov ${ }^{1,2}$, Evgeniy A. Losev², Denis A. Rychkov ${ }^{1,2}$, Adam L. Michaclchuk ${ }^{1,3}$, Ivana Lapsanska ${ }^{1,3}$, Colin R. Pulham

1. Novosibirsk State University

2. Institute of Solid State Chemistry and Mechanochemistry SB RAS

3. School of Chemistry, University of Edinburgh

email: eboldyreva@yahoo.com

Education tends to become more and more narrow. Knowledge about the world around and inside is being segmented into an ever-growing number of fields and sub-fields: mathematics, physics, chemistry, biology, planetary sciences, geology, mineralogy, and the list goes on. Not only are the humanities, sciences and arts taught separately, but there is also a growing trend not to teach all of these subjects to the same child. Instead, education is restricted to a narrow focus from an early age, limiting the breadth of skills and interests of any individual. As a result of this education technique, people tend to lose a general view of the world as a whole. Not only is this destructive for the individual, but places great limits on the development of society and culture.

Crystallography is a unique and fascinatingly multidisciplinary field that offers numerous links between the arts and sciences, between maths and chemistry, biology and physics. The objects of crystallography are beautiful, its language is strict and logical, yet open to intuition, and its applications are variable. Crystallography is, in some respects, a "meta-science", affording those that study it an integrated, harmonious vision of nature. As such, crystallography is an excellent subject to pursue for life-long education, and an exemplary discipline around which to focus public engagement efforts in both the sciences and arts.

In the present contribution we suggest a concept of using crystallography as a tool for a wholesome, balanced education that starts with children aged 3-6 years old and ends with seniors. At no point is a background in the sciences assumed. Some aspects of this concept have already been successfully tested in Novosibirsk, Russia, and in Edinburgh, UK. Others are yet to be implemented; we welcome volunteers to join us in this educational experiment. In particular, we discuss examples of communicating science to kids, of using crystallographic texts during French and English lessons. We also emphasise the co-teaching of crystallography and art, where the principles of each discipline are mutually reinforced. Hands-on sessions, drawing, and crystal growing can be ideally combined with the solving of mathematical problems, performing chemical and physical experiments, exploring natural objects (minerals, 